\title{
Early Emission of Core-Collapse Supernovae
}

\author{
Melina C. Bersten ${ }^{1}$, Omar Benvenuto ${ }^{2}$ and Ken'ichi Nomoto ${ }^{1}$ \\ ${ }^{1}$ Kavli Institute for the Physics and Mathematics of the Universe, Todai Institutes for \\ Advanced Study, University of Tokyo, 5-1-5 Kashiwanoha, Kashiwa, Chiba 277-8583, Japan \\ email: melina.bersten@ipmu.jp \\ ${ }^{2}$ Facultad de Ciencias Astronómicas y Geofísicas, Universidad Nacional de La Plata, Paseo del \\ Bosque S/N, B1900FWA La Plata, Argentina \\ email: obenvenu@f caglp.unlp.edu.ar
}

\begin{abstract}
We present a study of the early UV/Optical emission of the stripped-envelope supernovae based on a one-dimensional, Lagrangian model that solves the hydrodynamics and radiation transport in an expanding ejecta. The models are compared with observations to constrain the physical properties of the progenitor star, such as radius and mixing of radioactive nickel synthesized during the explosion. In particular, we present models for the early emission of the type IIb SN 2011dh and the Type Ib SN 2008D.
\end{abstract}

Keywords. supernovae: general, supernovae: individual (SN 2008D), supernovae: individual (SN 2011dh)

\section{Introduction}

Two main phases can be distinguished in a typical light curve (LC) of stripped-envelope supernovae (SE SNe, i.e. types IIb Ib and Ic): (a) the early UV/optical emission or "cooling phase" powered by the energy deposited by the shock wave and (b) a re-brightening to a "second peak" due to the decay of radioactive material synthesized during the explosion. Global properties of a SNe such as explosion energy $(E)$, ejected mass $\left(M_{\mathrm{ej}}\right)$, and the ${ }^{56} \mathrm{Ni}$ mass can be obtained by modeling the LC around the second peak. On the other hand, observations of the early emission provide unique information about the structure of the star previous to the explosion as well as the mixing process. The short duration of this early phase, specially for compact Wolf-Rayet stars, makes its observation elusive. However, in the last few years the early emission could be detected for a handful of objects and there is an increasing number of early observations, which will allow us to perform detailed studies. Here we present a study of the early SN emission using a one-dimensional, Lagrangian code that solves the hydrodynamics and radiation transport in an expanding ejecta (Bersten et al. (2011)). In particular, we analyze the case of the type Ib SN 2008D and the type IIb SN 2011dh which were observe at early times.

\section{Early emission models}

Early UV/optical emission is expected to occur after the arrival of the shock wave at the surface of the progenitor (shock break-out) and before the re-brightening due to the decay of radioactive material. This emission is a consequence of the nearly adiabatic cooling due to the expansion of the outermost layers of the ejecta. The duration of this early phase depends strongly on the size of the progenitor as shown in Fig. 1, where we present a set of LC models for progenitor radii of 2, 50, 100, 150, 200 and $270 R_{\odot}$. All these initial configurations have the same He core of $4 \mathrm{M}_{\odot}$ calculated from stellar evolution models (Nomoto \& Hashimoto 1988) plus a H-rich envelope smoothly attached to the 


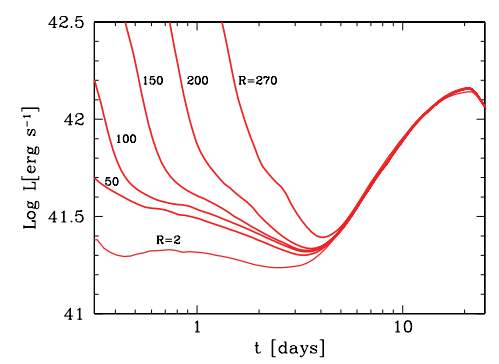

Figure 1. Bolometric LCs for models with different initial radii. The radius variation is accomplished by attaching essentially massless $\left(\lesssim 0.01 M_{\odot}\right)$ envelopes to the He4 model.
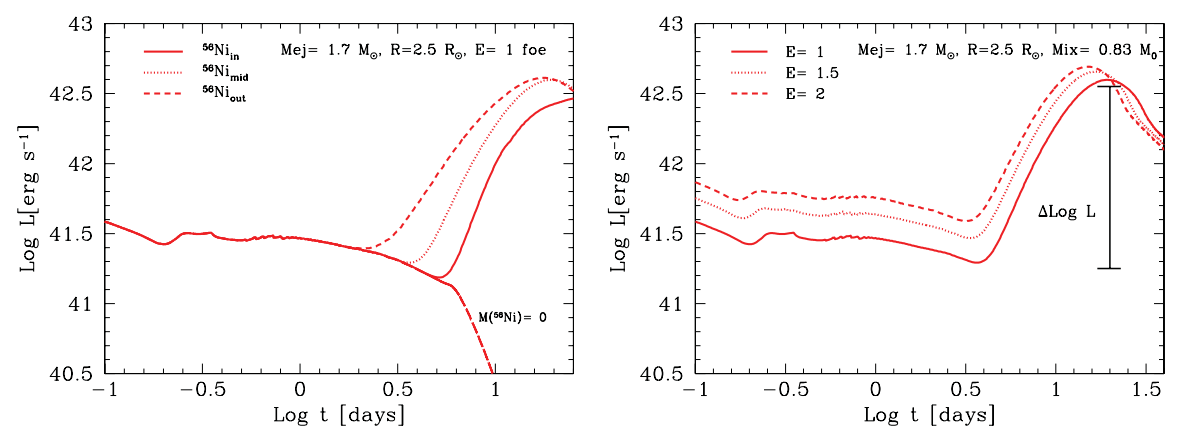

Figure 2. LCs for different ${ }^{56} \mathrm{Ni}$ mixing (left) and explosion energy (right).

the core to modify the progenitor structure. Clearly, the effect of the progenitor radius is only noticeable for times $\lesssim 5$ days (the exact epoch depends on the ${ }^{56} \mathrm{Ni}$ distribution). Observations before this epoch are required to discriminate between different progenitor radii. As previously noted by Dessart et al. (2011), models for compact progenitors show an initial plateau in the $\mathrm{LC}$ that is produced by He recombination. To test the dependence of the initial plateau on the ${ }^{56} \mathrm{Ni}$ mixing and explosion energy we calculated a set of hydrodynamical models for He core masses of 3 and $4 M_{\odot}$. Fig. 2 shows these results for He core mass of $3 M_{\odot}$. The models predict show that the initial plateau duration depends on the mixing of ${ }^{56} \mathrm{Ni}$ (left panel) but not on the energy (right panel). The luminosity of the plateau instead seems to depend mainly on the energy. For all the models we calculated the plateau duration $\left(\Delta t_{p}\right)$ and the difference in magnitude between plateau luminosity and main peak luminosity $(\Delta M)$. We found that the models predict $(1) \Delta t_{p}$ $\approx 4-10$ days, and $(2) \Delta M \approx 3$. Such values indicate that the initial plateaus can be detected in ongoing surveys. Note that the models presented here are helium-rich, so they should may be useful only for SNe Ib. We plan to extend our analysis to He-poor SNe.

\section{SN 2008D}

The type Ib SN 2008D attracted a good deal of attention because of its unusual observational features such as the detection of an X-ray transient (XRT) and an early optical light-curve peak. Here we present the first radiation-hydrodynamical models for the early emission of SN 2008D. We adopt a pre-SN model (from here on He8) with He core of 8 $M_{\odot}, R=1.4 R_{\odot}, E=8.4 \times 10^{51} \mathrm{erg} \mathrm{s}^{-1}$, and $M_{\mathrm{Ni}}=0.07 M_{\odot}$ similar to that assumed in Tanaka et al. (2009); (T09). Fig. 3 (right panel) shows the resulting LC for the He8 model (dashed line) as compared with the observations (Modjaz et al. 2009). Clearly, this model cannot explain the early emission shown by the observations. The difference 

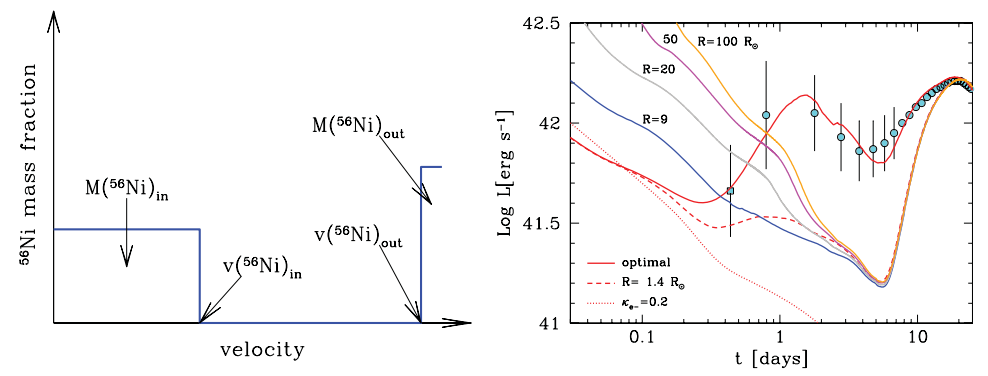

Figure 3. Left: Schematic doubly-peaked ${ }^{56} \mathrm{Ni}$ distribution. Right: Bolometric LC for model He8 with (red solid line) and without (red dashed line) external ${ }^{56} \mathrm{Ni}$ compared with the observations of SN 2008D. A model assuming a constant opacity of $\kappa_{e^{-}}=0.2 \mathrm{~g} \mathrm{~cm}^{-2}$ is also shown (red dot line). Models with larger radius (colors) cannot reproduce the early emission.

in luminosity between the hydrodynamical model and the observations at early times is $>0.5$ dex. In an attempt to improve the agreement with the observations, we artificially modified the radius of our pre-SN model by attaching nearly mass-less envelopes $(<0.01$ $M_{\odot}$ ) to the He8 model (see Fig. 3). However, we could not satisfactorily reproduce the early LC with these extended structures. To avoid this problem, we artificially placed some ${ }^{56} \mathrm{Ni}$ in the outermost layers of the ejecta in addition to the usual internal distribution, as schematically shown in the left panel of Fig. 3. A model based on He8 but with $\approx 0.01 M_{\odot}$ of ${ }^{56} \mathrm{Ni}$ at $v>20,000 \mathrm{~km} \mathrm{~s}^{-1}$ is shown with a red solid line in Fig. 3 . The agreement between this model and the observations is excellent.

\section{SN 2011dh}

The Type IIb SN 2011dh was discovered in the famous nearby galaxy M51. Soon after discovery, a source was identified as the possible progenitor of SN 2011dh in archival, multi-band HST images (Maund et al. 2011; Van Dyk et al. 2011). Photometry of the source was compatible with a yellow super-giant (YSG) star with $R \sim 270 R_{\odot}$. However, some authors have suggested a more compact progenitor $\left(R \sim R_{\odot}\right)$, claiming that the YSG star detected in the pre-SN images may be its binary companion or even an unrelated object (Arcavi et al. 2011; Van Dyk et al. 2011; Soderberg et al. 2011. In Bersten et al. (2012) we have used hydrodynamical models to show that an extended progenitor with $R \gtrsim 200 R_{\odot}$ in concordance with a YSG star is required to reproduce the early LC (see left panel of Fig 4). Our prediction has recently been confirmed by the reported disapearance of the YSG star (Van Dyk et al. 2013). In addition, modeling the LC around the second peak we found that a progenitor with He core mass of $\approx 4 M_{\odot}$, an explosion energy of $8 \times 10^{50} \mathrm{erg}$, and a ${ }^{56} \mathrm{Ni}$ mass of $0.063 M_{\odot}$ reproduces very well the observations. This optimal model (He4) is presented in Fig. 4 and it is consistent with a main-sequence mass of $\approx 13 M_{\odot}$. Furthermore, our models rule out progenitors with He core mass $>8 M_{\odot}$, implying that $M_{\mathrm{ZAMS}}<25 M_{\odot}$. Considering the limitations of single stars of such stellar masses to almost entirely expel the H-rich envelope via stellar winds, as required for SNe IIb, this result is indicative of a binary origin for SN 2011dh. In Benvenuto et al. (2013) we performed binary evolution calculations with mass transfer to reproduce the possible progenitor system of SN 2011dh. In Fig. 5 (left panel) we show the H-R digram for our prefered configuration of $16 M_{\odot}+10 M_{\odot}$ and an initial orbital period of 150 days. Note that the primary star ends its evolution at the right position as compared with the YSG star detected in the pre-SN images (red rectangle). Moreover, the final mass of the primary is $\approx 4 M_{\odot}$, which is consistent with our hydrodynamical modeling, and it includes a thin hydrogen envelope of $\approx 4 \times 10^{-3} M_{\odot}$ which is required 

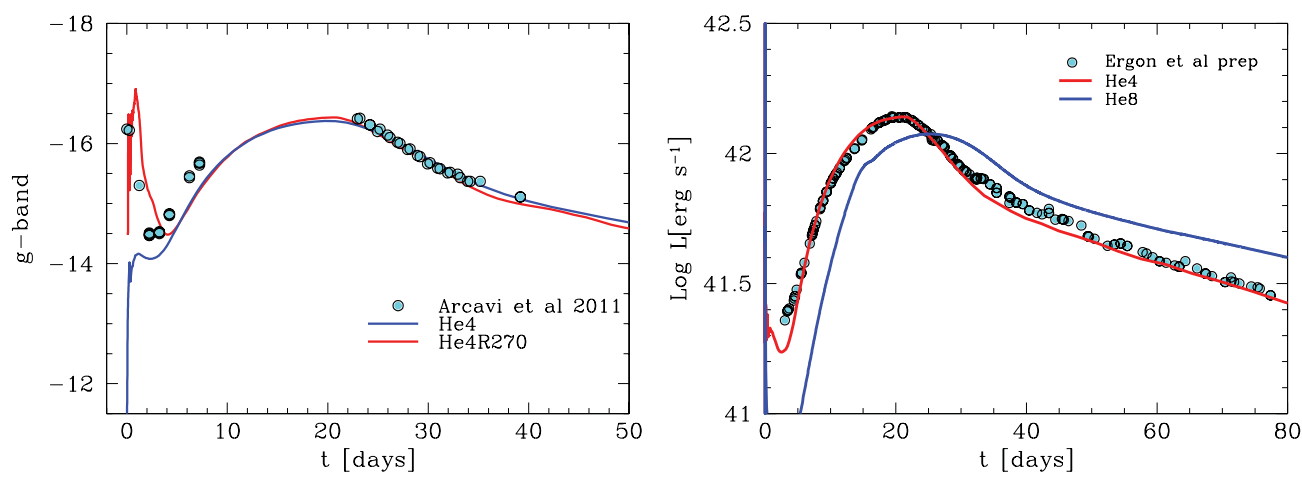

Figure 4. Observed and modeled $g^{\prime}$-band LCs (left panel) for the compact model He4 and the extended model. Clearly, the progenitor is required to be a supergiant in order to reproduce the observation. (Right panel) Bolometric LC for our optimal model, He4, with a He core mass of $\approx 4 M_{\odot}$ (red line) and for a model with He core mass of $8 M_{\odot}$ (He8; blue line) as compared with the observed bolometric LC of SN 2011dh (cyan dots). Note that the model with large helium core mass is not compatible with the observations.
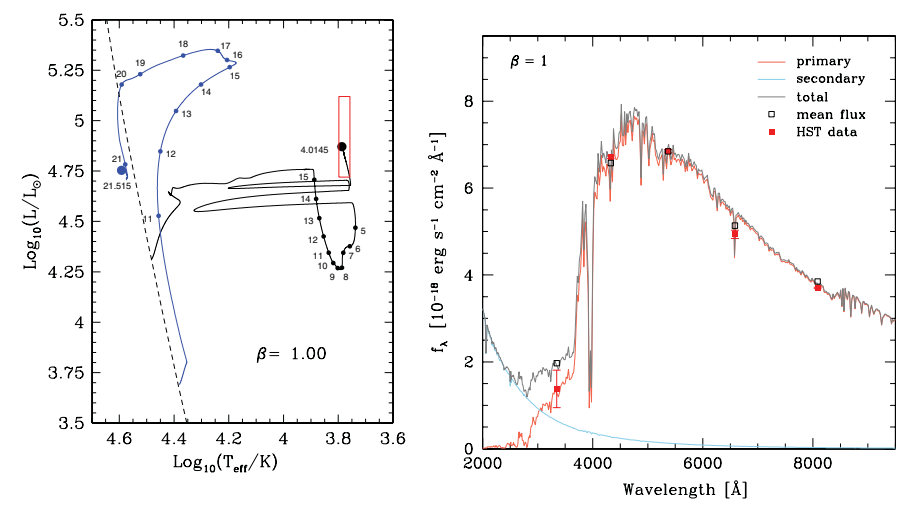

Figure 5. (Left panel) Evolutionary tracks in the H-R diagram for both components of a binary system with $M_{\mathrm{ZAMS}}$ of $16 M_{\odot}$ and $10 M_{\odot}$ and an initial period of 150 days. Labeled dots along the tracks indicate the masses of the stars (in solar units) while mass transfer by Roche-Lobe overflow occurs. (Right panel) Spectra of the donor and accreting stars (red and blue lines, respectively), and the sum of both (gray line). The mean synthetic (black squares) and observed fluxes (red squares) in each bandpass are shown.

to yield a SN IIb. The binary scenario was further tested by estimating the effect of the putative companion star on the pre-explosion photometry and comparing this with the observations (see Fig. 5, right panel). Because the secondary star is predicted to be an OB type star, the largest effect appears in the blue and UV range. The contribution of the secondary to the flux in the F336W band, however, is marginal, at the 1.5- $\sigma$ level for a conservative mass-transfer case, and at the 0.6- $\sigma$ level for a non-conservative case. The existence of the binary companion can be tested in the near future by a search for a blue object at the location of the SN.

\section{References}

Arcavi, I., Gal-Yam, A., Yaron, O., et al. 2011, ApJL, 742, L18

Benvenuto, O. G., Bersten, M. C., \& Nomoto, K. 2013, ApJ, 762, 74

Bersten, M. C., Benvenuto, O. G., Nomoto, K., et al. 2012, ApJ, 757, 31

Bersten, M. C., Benvenuto, O., \& Hamuy, M. 2011, ApJ, 729, 61 
Dessart, L., Hillier, D. J., Livne, E., et al. 2011, MNRAS, 414, 2985

Nomoto, K. \& Hashimoto, M. 1988, Phys. Rep., 163, 13

Maund, J. R., Fraser, M., Ergon, M., et al. 2011, ApJL, 739, L37

Modjaz, M., Li, W., Butler, N., et al. 2009, ApJ, 702, 226

Soderberg, A. M., Margutti, R., Zauderer, B. A., et al. 2011, arXiv:1107.1876

Tanaka, M., Tominaga, N., Nomoto, K., et al. 2009, ApJ, 692, 1131

Van Dyk, S. D., Filippenko, A. V., Fox, O., et al. 2013, ATel 4850

Van Dyk, S. D., Li, W., Cenko, S. B., et al. 2011, ApJL, 741, L28

\section{Discussion}

CAPPELlaro: What are the observations where you have evidence for a blue companion for the progenitor of SN in M51

Bersten: I used the model information, T- effective and L for the primary and secondary stars, and calculated the SED of each star using Atmospheric models available in the literature. Then I calculated the composite spectra and the mean fluxes in the same photometric band and then compared with the pre-explosion observations. The blue companion is only marginally detected (1.4 sigma) in the Bluest observation band. 